\title{
The use of continuous monitoring blood culture systems in the diagnosis of catheter related sepsis
}

\author{
M S Rogers, B A Oppenheim
}

\begin{abstract}
Aim-To assess whether the information provided by automated continuous monitoring blood culture systems could aid in the diagnosis of catheter related sepsis.

Methods-Serial dilutions of a strain of coagulase negative staphylococcus were inoculated into the BacT/Alert blood culture system. Blood culture results for seven patients with possible catheter related sepsis from coagulase negative staphylococci were reviewed.

Results-Time to positivity and length of lag period were strongly related to the concentration of bacteria inoculated (average decrease of 1.5 hours to positivity for each 10-fold increase in concentration). Time to positivity and length of lag period were significantly shorter for central line blood cultures than for those taken from peripheral sites.

Conclusions-Using data already measured by continuous monitoring blood culture systems may provide a simple alternative to quantitative blood cultures for the diagnosis of catheter related sepsis. (f Clin Pathol 1998;51:635-637)
\end{abstract}

Keywords: catheter related sepsis; blood cultures

Despite the widespread use of intravascular catheters, the diagnosis of catheter related sepsis remains problematic. Many of the diagnostic methods available require the removal of the line to access the catheter tip, ${ }^{1-3}$ and this is not always practical. An endoluminal brush method has been evaluated, ${ }^{4}$ but this involves additional expense and there are concerns about dislodging potential pathogens into the circulation. Differential quantitative blood cultures have been suggested as an alternative to removing the catheter for the diagnosis of catheter related sepsis. ${ }^{5-8}$ However, quantitative blood cultures are extremely labour intensive to perform and great efforts have to be made to avoid contamination of the cultures. Since many laboratories now use automated continuous monitoring blood culture systems, we attempted to assess whether the information provided by these could aid in the diagnosis of catheter related sepsis.

Withington Hospital, West Didsbury, Manchester M20 2LR, UK

M S Rogers

B A Oppenheim

Correspondence to: Dr Oppenheim.

Accepted for publication 16 April 1998

Methods

BACTERIAL STRAIN

A strain of coagulase negative staphylococcus isolated from a blood culture was used for the bacterial load studies.
BLOOD CULTURE SYSTEM

The BacT/Alert (Organon Teknica, Durham, North Carolina, USA) continuous monitoring system was used. ${ }^{9}$ For the bacterial loading studies standard aerobic and anaerobic blood culture bottles were used. Most blood cultures from patients were received in pairs of aerobic and anaerobic culture bottles, but in some cases the FAN aerobic bottles were used to enhance the recovery of fastidious organisms.

BACTERIAL LOADING STUDIES

Following overnight incubation in nutrient broth, the strain of coagulase negative staphylococcus was sequentially diluted into sterile nutrient broth in order to obtain the chosen dilutions. These dilutions were confirmed using Miles and Misra counts for both aerobic and anaerobic culture. One millilitre of each study dilution was added to $9 \mathrm{ml}$ of defibrinated horse blood and then inoculated into both aerobic and anaerobic blood culture bottles. These were immediately inserted into the BacT/Alert system and incubated for five days.

\section{PATIENT DATA ANALYSIS}

We reviewed blood culture records for patients who had an indwelling central venous catheter for the period January to May 1997, identifying cases where coagulase negative staphylococci had been isolated from both peripheral and central blood cultures and where no other source of infection was identified (possible catheter related sepsis). Those that were positive on receipt by the laboratory were not used for this analysis.

DATA INTERPRETATION AND STATISTICAL METHODS

The growth pattern for each individual blood culture bottle for both in vitro and patient cultures was analysed in terms of time to positivity (hours), length of lag phase ( $\mathrm{mm}$ ), angle of lag line to $\mathrm{Y}$ axis, angle of lag line to $\mathrm{X}$ axis, and angle between extrapolated lag line and log line (fig 1).

The relations in the bacterial loading studies were investigated using least squares regression methods with concentrations on a logarithmic scale. The patient data analyses used a paired $t$ test approach to analysis of variance. The analyses were all performed in GLIM. ${ }^{10}$

\section{Results} . In the bacterial load studies there was a strong and highly significant $(p<0.001)$ straight line relation between time to positivity and the logarithm of 


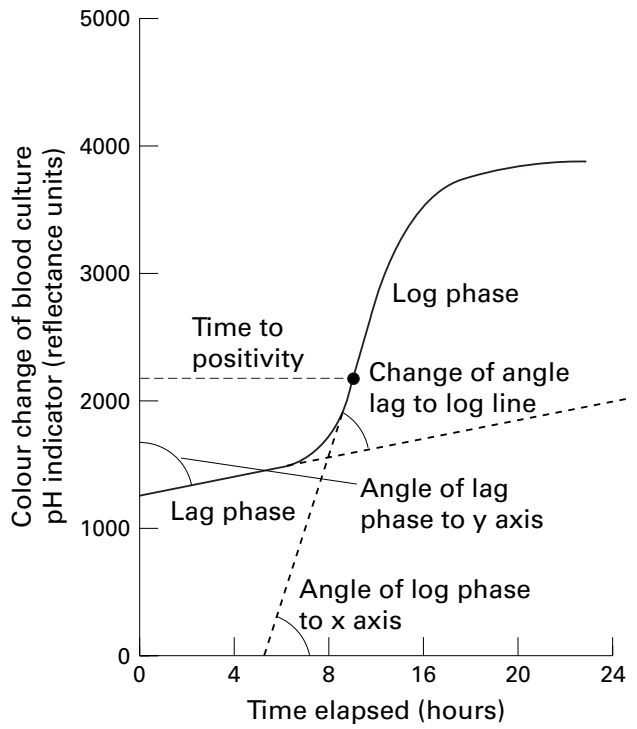

Figure 1 Graph of colour change of blood culture $\mathrm{pH}$ indicator showing indices measured for both in vitro and patient cultures.

the initial bacterial concentration and this was the same for aerobic and anaerobic bottles. The slope of the trend lines modelling this relation showed that there was, on average, a decrease

Table 1 Indices measured in bacterial load studies using seven different dilutions of organism

\begin{tabular}{|c|c|c|c|c|c|c|}
\hline Bacteria/ml & $\begin{array}{l}\text { Bottle } \\
\text { type }\end{array}$ & $\begin{array}{l}\text { Time to } \\
\text { positivity } \\
\text { (h) }\end{array}$ & $\begin{array}{l}\text { Length of } \\
\text { lag phase } \\
(\mathrm{mm})\end{array}$ & $\begin{array}{l}\text { Angle of lag } \\
\text { line to } Y\left({ }^{\circ}\right)\end{array}$ & $\begin{array}{l}\text { Angle of log } \\
\text { line to } X \\
()^{\circ}\end{array}$ & $\begin{array}{l}\text { Angle of change } \\
\text { log to lag line }(\odot)\end{array}$ \\
\hline 10000 & AER & 12.3 & 1.35 & 77 & 77 & 63 \\
\hline 10000 & AN & 12.5 & 1.30 & 81 & 72.5 & 64 \\
\hline 1000 & AER & 13.5 & 1.45 & 80 & 75 & 66 \\
\hline 1000 & $\mathrm{AN}$ & 13.5 & 1.45 & 81 & 71 & 62 \\
\hline 500 & AER & 14.0 & 1.55 & 79 & 76 & 65 \\
\hline 500 & AN & 14.0 & 1.55 & 80 & 76 & 67 \\
\hline 100 & AER & 14.7 & 1.65 & 78 & 74 & 66 \\
\hline 100 & AN & 14.8 & 1.75 & 77 & 76 & 62 \\
\hline 50 & AER & 15.3 & 1.80 & 79 & 77 & 67 \\
\hline 50 & AN & 15.5 & 1.75 & 79 & 75 & 64 \\
\hline 10 & AER & 17.2 & 1.85 & 79 & 75 & 63 \\
\hline 10 & AN & 16.3 & 2.05 & 78 & 77 & 67 \\
\hline 5 & AER & 17.7 & 2.05 & 80 & 76 & 65 \\
\hline 5 & AN & 17.2 & 2.15 & 77 & 77 & 64 \\
\hline
\end{tabular}

AER, aerobic; $\mathrm{AN}$, anaerobic.

Table 2 Indices measured in seven patients with probable catheter related sepsis

\begin{tabular}{|c|c|c|c|c|c|c|c|}
\hline Patient & $\begin{array}{l}\text { Site of } \\
\text { sample }\end{array}$ & $\begin{array}{l}\text { Bottle } \\
\text { type }\end{array}$ & $\begin{array}{l}\text { Time to } \\
\text { positivity } \\
\text { (h) }\end{array}$ & $\begin{array}{l}\text { Length of } \\
\text { lag phase } \\
(\mathrm{mm})\end{array}$ & $\begin{array}{l}\text { Angle of } \\
\text { lag line to } \\
Y()\end{array}$ & $\begin{array}{l}\text { Angle of } \\
\text { log line to } \\
X(\odot)\end{array}$ & $\begin{array}{l}\text { Angle of } \\
\text { change log to } \\
\text { lag line (O) }\end{array}$ \\
\hline A & $\mathrm{CEN}$ & $\mathrm{AN}$ & 13.0 & 1.5 & 68 & 79 & 57 \\
\hline A & CEN & AER & 12.5 & 1.4 & 74.5 & 81 & 67 \\
\hline A & PER & $\mathrm{AN}$ & 13.8 & 1.6 & 71.5 & 74 & 56 \\
\hline A & PER & AER & 13.8 & 1.7 & 69.5 & 79 & 59 \\
\hline B & CEN & AN & 13.7 & 1.8 & 78 & 78 & 66 \\
\hline B & CEN & AER & 13.5 & 1.6 & 81 & 78 & 69 \\
\hline B & PER & AN & 20.5 & 2.6 & 78.5 & 61 & 50 \\
\hline B & PER & AER & 18.8 & 2.3 & 82.5 & 73 & 64 \\
\hline C & CEN & $\mathrm{AN}$ & 11.8 & 1.3 & 71 & 72 & 54 \\
\hline C & CEN & AER & 11.8 & 1.3 & 71 & 69 & 51 \\
\hline C & PER & $\mathrm{AN}$ & 20.0 & 2.5 & 71.5 & 53 & 36 \\
\hline C & PER & AER & 20.5 & 2.6 & 70 & 61 & 43 \\
\hline D & CEN & FA & 9.0 & 1.1 & 63 & 80 & 55 \\
\hline D & PER & FA & 10.7 & 1.3 & 62 & 82 & 54 \\
\hline $\mathrm{E}$ & CEN & AN & 7.3 & 0.5 & 67 & 65 & 40 \\
\hline E & CEN & AER & 7.7 & 0.7 & 67 & 76 & 52 \\
\hline E & PER & AN & 11.2 & 1.2 & 73 & 71 & 55 \\
\hline E & PER & AER & 12.0 & 1.35 & 71.5 & 75 & 58 \\
\hline F & CEN & AER & 8.7 & 1.0 & 70 & 71 & 52 \\
\hline $\mathrm{F}$ & PER & AN & 7.2 & 0.65 & 65 & 74 & 50 \\
\hline G & CEN & AER & 7.0 & 0.5 & 68 & 69 & 49 \\
\hline G & PER & AN & 7.0 & 0.6 & 66.5 & 79 & 56 \\
\hline
\end{tabular}

AER, aerobic; AN, anaerobic; CEN, central; FA, FAN aerobic bottle for fastidious organisms; PER, peripheral. of 1.5 hours ( $95 \%$ confidence interval 1.35 to 1.69) in the time to positivity for each 10 -fold increase in concentration. The length of the lag period was also strongly related to concentration of bacteria inoculated, and the slope was virtually the same for aerobic and anaerobic bottles. The angle of the lag line with the vertical appeared to be related, positively, to initial concentration in the anaerobic bottles, but not in the aerobic. The estimated change in angle per 10 -fold increase in concentration for the anaerobic bottles was 1.3 degrees (0.6 to 1.9$)$. There was a slight negative trend in the aerobic bottles, but not significantly different from zero. The angle of the log line to the horizontal followed the same pattern as the angle of the lag line to the vertical and was only related to the initial concentration in the anaerobic bottles although in this case the relation was negative. The estimated change in this angle per 10-fold increase in the initial concentration was a decrease of 1.6 degrees (0.6 to 2.6).

We identified seven patients who fulfilled the criteria for possible catheter related sepsis (no patients were identified where there were simultaneous positive catheter tip samples) and who had at least one set of central and peripheral cultures taken simultaneously. Time to positivity in these patients was the same, apart from chance differences, whether aerobic or anaerobic bottles were used. However, time to positivity for peripheral sample cultures was apparently longer than for central line sample cultures, suggesting that the inoculum in central line specimens was higher than in peripheral site specimens. This varied from patient to patient, so generalisations should be made cautiously. However, the time to positivity for central line sample cultures were significantly shorter than for peripheral sample cultures by, on average, 4.1 hours (2.4 to 5.8). The length of the lag period followed much the same pattern, as this reflects the time to positivity. The angle of the lag line to the vertical, the angle of the log line to the horizontal, and the angle between the two lines were not significantly different with respect to sampling site or bottle type.

\section{Discussion}

The bacterial load studies suggest that, certainly with the BacT/Alert system, time to positivity and length of lag phase are directly related to initial inoculum. In these studies, the concentration of bacteria was the only variable in the samples as the tests were performed under strictly controlled conditions and each sample was treated identically. Other factors known to affect bacterial lag phase such as temperature and metabolic conditions seem not to be relevant in this case.

In patient samples, the time to positivity and length of lag phase were shorter for the cultures taken though the central line, suggesting that there were larger numbers of bacteria in these inocula. However, these results refer only to patients with coagulase negative staphylococcal bacteraemias and they may not be applicable to more fastidious or slower growing organisms. It is of interest that in some cases time to positivity for patient samples was shorter than in the 
bacterial loading studies, despite the high inocula used. This almost certainly reflects preincubation on ward or pathology areas before insertion of the bottles into the BacT/ Alert system.

If we are to be able to evaluate novel or more effective treatments for central line infections, it is essential to have an accurate diagnostic method which does not require sacrifice of the line. Using data already available in continuous monitoring blood culture systems is attractive because it requires no additional technical work or costs. Our preliminary data require further rigorous evaluation against established diagnostic methods.

We are grateful to the PHLS Statistics Unit for statistical advice

1 Maki DG, Weise CE, Sarafin HW. A semiquantitative culture method for identifying intravenous-catheter-related infection. N Engl f Med 1977;296:1305-9.
2 Cleri DJ, Corrado ML, Seligman SJ. Quantitative culture of intravenous catheters and other intravascular inserts. $f$ Infect Dis 1980;141:781-6.

3 Cooper GL, Hopkins CC. Rapid diagnosis of intravascular catheter-associated infection by direct Gram staining of catheter segments. N Engl f Med 1985;312:1142-7.

4 Kite P, Dobbins BM, Wilcox MH, et al. Evaluation of a novel endoluminal brush method for in situ-diagnosis of catheter related sepsis. F Clin Pathol 1997;50:278-82.

5 Wing EJ, Norden CW, Shadduck RK, et al. Use of quantitative bacteriologic techniques to diagnose catheter-related sepsis. Arch Intern Med 1979;139:482-3.

6 Raucher HS, Hyatt AC, Barzilai A, et al. Quantitative blood cultures in the evaluation of septicemia in children with Broviac catheters. F Pediatr 1984;104:29-33.

7 Weightman NC, Simpson EM, Speller DCE, et al. Bacteraemia related to indwelling central venous catheters: prevention, diagnosis and treatment. Eur f Clin Microbiol Infect Dis 1988;7:125-9.

8 Quilici N, Audibert G, Conroy M-C, et al. Different quantitative blood cultures in the diagnosis of catheter-related (2) 70 .

9 Thorpe TC, Wilson ML, Turner JE, et al. BacT/Alert: an automated colorimetric microbial detection system. $\mathcal{F}$ Clin Microbiol 1990;28:1608-12.

10 Francis B, Green M, Payne C, eds. The GLIM system release 4 manual. Oxford: Oxford University Press, 1993. 